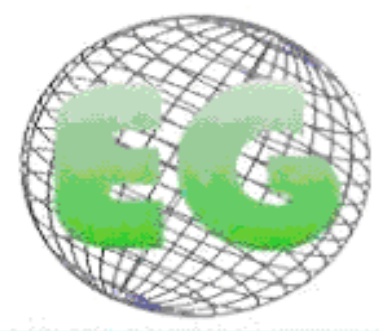

ISSN 1695-6141 N'25
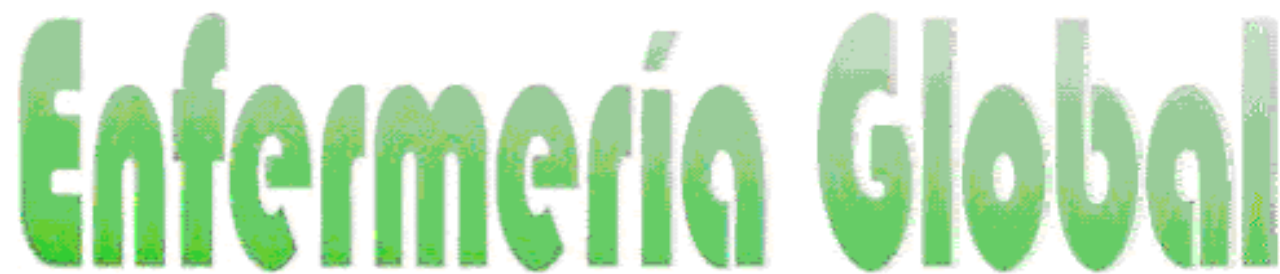

Revista electrónica trimestral de Enfermería

Enero 2012

www.um.es/egloball

\title{
Efecto de un sistema automático de dispensación de medicamentos sobre el gasto farmacéutico y el grado de satisfacción del usuario
}

An automatic dispensing system medications effect in pharmaceutical expenditure and satisfaction user level

\section{*Zafra Fernández, J. L., "Isla Tejera, B., "Del Prado Llergo, J.R.}

*U. G. C. de Farmacia Hospitalaria. Hospital Universitario Reina Sofía. Córdoba.

Palabras clave: Sistemas automáticos de dispensación de medicamentos; botiquín de planta; Unidad de Cuidados Intensivos; gasto farmacéutico; política de consumo; satisfacción usuarios.

Keywords: Automatic dispensing medications; plant kit; Intensive Care Unit; pharmaceutical expenditure; consumer policy; user satisfaction.

\section{RESUMEN}

Objetivo: Evaluar en términos económicos el efecto de la sustitución de botiquines tradicionales por la implantación de Sistemas Automáticos de Dispensación de Medicamentos en la Unidad de Cuidados Intensivos. Analizar el grado de aceptación de dicho cambio por parte de los usuarios.

Método: Para el análisis económico, se consideraron los costes directos e indirectos tangibles, derivados de la implantación: inversión inicial de capital, coste del personal implicado, coste en política de consumo de medicamentos. Todos estos aspectos se evaluaron antes y después de la implantación. El grado de satisfacción de los usuarios se evaluó a través de un cuestionario estandarizado.

Resultados: Tras la estimación de los costes, comprobamos que el gasto realizado en la inversión inicial se verá rápidamente compensado fundamentalmente por el coste referido a la política de consumos de medicamentos con una reducción del $24 \%$ con respecto al sistema de dispensación anterior. El coste en el capitulo de personal también se ve reducido un $11 \%$, así como el coste/estancia y coste/ingreso que disminuyen un $26 \%$ y $30 \%$ respectivamente en relación al sistema anterior. La evaluación del cuestionario reveló que los usuarios están satisfechos con la implantación y un $84 \%$ del personal de enfermería lo recomendaría a otras unidades.

Conclusiones: Los Sistemas Automáticos de Dispensación de Medicamentos constituyen una nueva herramienta tecnológica para el control del gasto farmacéutico, con buena aceptación por parte de los usuarios. 


\title{
ABSTRACT
}

Objective: To evaluate in economic terms, the effect of replacing traditional kits for the implementation of automated dispensing of medications in the Intensive Care Unit. Analyze the degree of acceptance of such changeon the part of users.

\begin{abstract}
Method: For the economic analysis is considered tangible direct and indirect costs resulting from the implementation: initial capital investment, cost of staff involved, the political cost of drug consumption. All these aspects were evaluated before and after implantation. The degree of user satisfaction was assessed using a standardized

questionnaire.

Results: After the estimation of costs, we found that the expenditure incurred on the initial investment will be quickly offset by the cost mainly referred to the politics of consumption of drugs with a reduction of $24 \%$ over the previous delivery system. The cost in personal chapter is also reduced by $11 \%$, and the cost per stay and cost / income fell by $26 \%$ and $30 \%$ respectively over the previous system. The evaluation questionnaire revealed that users are satisfied with the implementation and $84 \%$ of nurses would recommend it to other units.
\end{abstract}

Conclusions: Automated Dispensing Systems Drugs are a new technological tool to control drug spending, with good acceptance by users.

\section{INTRODUCCIÓN}

En los últimos 20 años, el importante avance en el desarrollo de tecnologías aplicadas al ámbito sanitario ha contribuido a incrementar el grado de fiabilidad y calidad de los sistemas logísticos relacionados con las actividades asistenciales ${ }^{1,2,3,4}$. En el área del medicamento, ha sido clave la incorporación de la robótica, la automatización y la informática a todo el proceso del dispensación y uso de los medicamentos incrementando la eficiencia, la calidad y la seguridad de la farmacoterapia del paciente- Un ejemplo ha sido el desarrollo de Sistemas de Dispensación Automatizada de Medicamentos (SADME), cuyo uso ha permitido mejorar la logística de distribución de medicamentos al disminuir el tiempo utilizado por el equipo de salud y de mejorar la eficacia y seguridad de esta tarea al disminuir los errores de medicación inherentes al proceso de distribución. Así mismo, esta tecnología facilitaría tanto la gestión de la información relacionada con la prescripción de medicamentos para los enfermos como la optimización de los costes directos e indirectos asociados ${ }^{5}$.

Los SADME constituyen una red de estaciones de almacenamiento seguro localizado en las áreas de atención a pacientes. Cada estación está controlada por su propio microprocesador y funciona de forma similar a los cajeros automáticos de un banco, conectados a un sistema de procesamiento central, ubicado generalmente en el Servicio de Farmacia (SF). Para obtener medicamentos de la estación, los usuarios autorizados acceden a ella y en ese momento queda registrada automáticamente toda la información de la transacción, incluido el nombre del paciente, sus datos clínicos, nombre del usuario y cantidad que debe ser retirada, con fines contables, de reabastecimiento y de facturación. Estos dispositivos se utilizan como complemento del sistema tradicional de medicamentos en dosis unitaria en aquellas unidades donde no sea posible individualizar la dispensación por paciente, bien por el alto grado de rotación de los pacientes, o bien porque los cambios en el tratamiento se producen con mucha frecuencia y a menudo de forma urgente. Es el caso de áreas como Medicina Intensiva, Quirófano, Observación, Urgencias, etc. ${ }^{6}$

Lo que diferencia este sistema automático de dispensación de medicamentos de los sistemas tradicionales de inventario y distribución de medicamentos es que permite a la farmacia, no sólo almacenar y llevar el seguimiento del inventario en la Unidad Clínica, sino además conocer la farmacoterapia del paciente. En este contexto es donde los SADME están demostrando ser beneficiosos como herramienta de gestión clínica ${ }^{7}$. 
En un escenario donde la limitación económica puede ser un elemento determinante para el desarrollo de cualquier proyecto, es imprescindible evaluar las intervenciones sanitarias que se realicen. Por tanto, si se quiere maximizar el bienestar social, es preciso considerar tanto los beneficios como los costes, que afectan directa o indirectamente a la asignación de recursos $^{8}$. En la actualidad las ventajas clínicas y económicas que para el sistema de salud supone la utilización de sistemas automatizados de medicamentos, así como la aceptación y convencimiento del personal del equipo de salud sobre la validez del sistema, explican tanto la credibilidad alcanzada entre los usuarios como la actual estabilidad del coste de medicamentos, tal y como se documenta en la bibliografía. ${ }^{9,10,11,12}$

Antes de la implantación de los SADME en nuestro hospital, la Unidad de Cuidados Intensivos (UCI) tenía un sistema tradicional de distribución y almacenamiento que constaba de un almacén central de farmacia para la Unidad y pequeños botiquines en cada módulo de UCl. Dos veces por semana, enfermería generaba un pedido de reposición de botiquín que el SF realizaba y entregaba en la Unidad, además de la entrega de pedidos urgentes. Una vez en la planta de hospitalización, el personal de enfermería de la Unidad se encargaba de su almacenamiento y distribución por los distintos módulos.

Para la implantación de los SADME, se estableció un cronograma en el que se detalló la fecha de instalación de los seis sistemas automáticos de dispensación de medicamentos $\left(\right.$ Omnicell $\left.{ }^{\circledR}\right)$.

Durante un periodo de 48-72h coexistieron ambos sistemas. La frecuencia de reposición de medicamentos está supeditada a la configuración establecida para los SADME, y a la parametrización efectuada en relación al volumen almacenado y a la rotación de medicamentos en cada módulo. En nuestro caso, tres veces por semana, se imprime de forma automática un listado con los medicamentos necesarios para la reposición normal de cada uno de los armarios y diariamente se imprime el listado de reposición urgente para aquellos medicamentos cuyo stock se encuentra por debajo del stock de seguridad, la reposición en ambos casos siempre es hasta completar el stock máximo del medicamento. Una vez preparado el pedido en el SF, la misma persona se encarga de reponerla en los armarios.

De este modo, en este trabajo nos propusimos analizar el efecto que la implantación de los SADME ha tenido en nuestro hospital, en materia económica y el grado de aceptación de los usuarios.

\section{MÉTODOS}

\section{Población y variables de estudio}

Se evaluó el efecto de la implantación de los SADME (Omnicell ${ }^{\circledR}$. Palex Medical S.A. División Ingeniería Hospitalaria Francesc Viñas, Barcelona. España) en UCl en un periodo de 5 meses, de abril a agosto 2009, frente al mismo periodo de 2010, que comprenden los periodos pre y post-instalación. Se analizaron variables económicas tras ambos periodos y el grado de satisfacción de los usuarios tras su implantación. Los usuarios fueron los farmacéuticos, enfermeras, auxiliares de enfermería, auxiliares administrativos y celadores encargados de las diferentes funciones asociadas a ambos circuitos de trabajo. [Figura 1] 


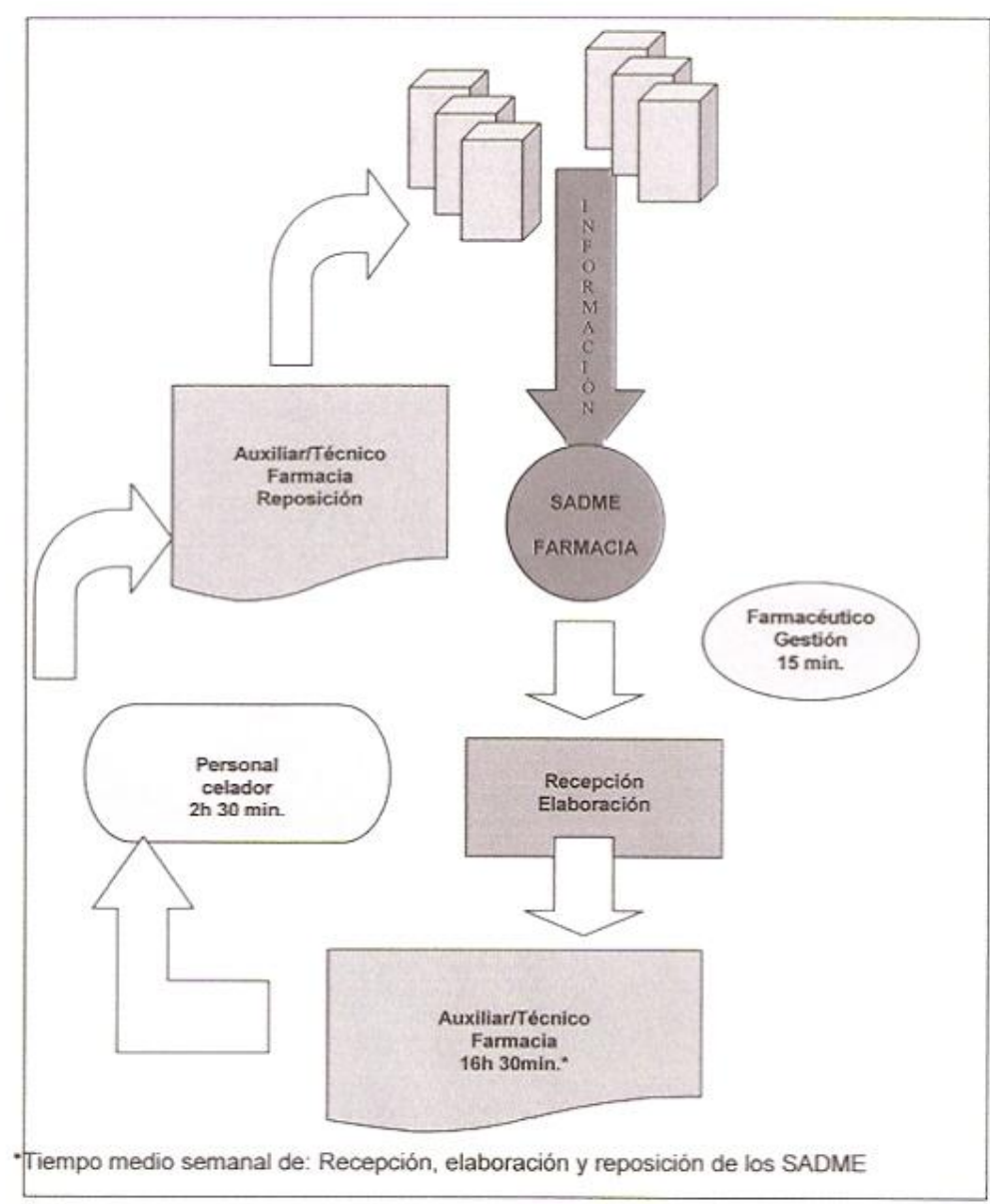

\section{Indicadores Económicos}

Para la evaluación económica se han considerado las siguientes fases:

-Identificación de los factores o activos que van a verse afectados.

-Cuantificación de cada uno de los costes considerados.

-Temporalidad: No se tuvo en cuenta la aplicación de una tasa de descuento en los costes por tratarse de un periodo no superior al año.

Se han determinado los costes directos e indirectos tangibles, positivos y negativos que pueden ser valorados de forma explicita a través del sistema de precios de mercado:

a) Coste de los SADME adquiridos mediante de concurso público.

b) Coste del personal implicado en cada una de las alternativas; para su análisis se ha recogido durante un mes los tiempos utilizados por los facultativos, enfermería, auxiliares de clínica, auxiliares de farmacia/técnicos en farmacia, auxiliares administrativos y celadores, en cada una de las alternativas analizadas. Estos tiempos incluyen todos los procesos sanitarios que garantizan la disponibilidad de medicamentos para su administración en $\mathrm{UCl}$, así como los procesos de control y asignación de los costes subsidiarios al 
mismo, y que forman parte intrínseca del proceso global de distribución de medicamentos. Los costes por unidad temporal de trabajo para cada uno de los diferentes colectivos sanitarios que intervienen en las alternativas fueron obtenidos a partir de las retribuciones de personal publicados por el Sistema Andaluz de Salud (S.A.S) para el ejercicio 2009 (Resol. 0063/09 (23-3)1.

c) Costes de la política de consumo de medicamentos; se ha realizado el calculo del consumo de medicamentos en $\mathrm{UCl}$, el gasto de medicamentos en base a la diferencia de actividad: coste /estancia y coste/ingreso.

\section{Grado de Satisfacción}

El grado de satisfacción de los usuarios se evaluó a través de un cuestionario (Anexo I) que incluía datos demográficos, laborales y profesionales.

a) Población de estudio: la población diana se corresponde con los usuarios reales del SADME, siendo el método de muestreo no probabilístico, al incluir a todo el personal de enfermería y auxiliares del servicio. La encuesta se realizó siempre de manera autoadministrada.

b) Valoración del Grado de Satisfacción: las variables utilizadas en el cuestionario como determinantes de la percepción e intención de uso han sido validadas en anteriores investigaciones y se han seleccionado a partir del modelo TBP (Theory of Planned Behavior) donde se afirma que el determinante más importante de la conducta son las intenciones.

c) Análisis estadístico: a la hora de estimar el tamaño muestral se tuvo en cuenta la proporción esperada de los usuarios que considerarían útil la implantación de estos sistemas, considerando que para un índice de confianza del $95 \%$ y un error del 5\% necesitaríamos una muestra de 75 usuarios. Como variable dependiente se consideró el grado de satisfacción global del usuario con estos sistemas. Para facilitar el análisis de los resultados, las respuestas medidas en escala de Likert del 1 a 7 , fueron dicotomizadas en positiva y negativa, considerando como actitud neutra el valor de 4 (mediana en la escala): las conductas positivas con valores inferiores al neutro y el resto de valores de la escala como negativo. Se realizó un estudio descriptivo para las variables cualitativas, expresándolas como frecuencias absolutas y porcentuales. Para las variables cuantitativas se utilizó la media aritmética, desviación típica y rango. En el análisis bivariante, se realizaron los test paramétricos y no paramétricos oportunos con de tablas de contingencia. Todos los contrastes fueron bilaterales y se consideraron significativos aquellos con una $p \leq 0,05$. Para el análisis se utilizó el programa SPSS v.17.0.

\section{RESULTADOS}

\section{Económicos}

El capital invertido en los SADME instalados en UCI fue de $231.000 €$, los armarios se adquirieron por concurso público, gracias a la aprobación del proyecto por parte de la Agencia de Evaluación de Tecnologías Sanitarias de Andalucía, SAS. 
El coste del personal implicado en cada una de las alternativas fue de $5.747,0 €$ para el botiquín tradicional frente a 5.098,0€ del sistema SADME, tal y como se detalla en la tabla I, el coste total del personal se reduce, obteniéndose un beneficio en este capitulo del $11 \%$. El personal que ve más reducidas sus horas de trabajo destinadas a labores de logística y de gestión de botiquines es el personal de enfermería de la Unidad Clínica, supervisora, y especialmente auxiliares de enfermería de la Unidad. Por el contrario, el tiempo empleado por el personal auxiliar del SF para preparar los pedidos ordinarios de la Unidad se ve considerablemente aumentado, al encargarse también de la reposición, control de caducidades y mantenimiento de los SADME, pasando de 144 horas a 792 horas anuales.

Tabla I.- Costes indirectos personal para los dos sistemas de distribución.

\begin{tabular}{|c|c|c|c|c|c|c|c|c|}
\hline \multirow[b]{2}{*}{ Profesional } & \multicolumn{4}{|c|}{ Botiquín tradicional } & \multicolumn{2}{|l|}{ SADME } & \multirow[b]{2}{*}{ diferencia } & \multirow[b]{2}{*}{$\%$} \\
\hline & horas/año & coste/hora & coste/personal & horas año & coste/hora & coste/personal & & \\
\hline Farmacéutico & 12 & 13,8 & 165,0 & 12 & 13,8 & 165,0 & 0,0 & $0 \%$ \\
\hline Supervisor & 120 & 9,6 & 1.154 & 0 & 9,6 & 0,0 & 1.154 & $-100 \%$ \\
\hline Auxiliar & 576 & 5,5 & $3.154,9$ & 0 & 5,5 & 0,0 & $3.154,9$ & $-100 \%$ \\
\hline Administrativo & 36 & 5,5 & 198,8 & 0 & 5,5 & 0,0 & 198,8 & $-100 \%$ \\
\hline Tèc/Aux Farm & acia 144 & 5,5 & 788,7 & 792 & 5,5 & $4.338,0$ & $-3.549,3$ & $450 \%$ \\
\hline Celador & 57,6 & 5,0 & 285,4 & 120 & 5,0 & 594,6 & $-309,2$ & $108 \%$ \\
\hline Total & 945,6 & 44,8 & $5.747,0$ & 924 & 44,8 & $5.098,0$ & 649,0 & $-11 \%$ \\
\hline
\end{tabular}

En cuanto al cálculo de los costes asociados al consumo de medicamentos, en la tabla II se describe el consumo en $\mathrm{UCl}$, la relación coste/ingreso y coste/estancia. Como puede observarse, se ha producido una reducción neta del consumo de 205.607€ en este periodo, lo que supone una disminución del gasto en medicamentos del $24,21 \%$. Disminuyendo el coste en medicamentos por ingreso y estancia en un $30 \%$ y $26 \%$ respectivamente.

Tabla II. Costes directos derivados del consumo farmacéutico en UCI.

\begin{tabular}{ccccc}
\hline & 2009 & 2010 & & \\
(abril-agosto) & (abril-agosto) & 727 & 58 & Diferencia \\
\hline Ingresos & 669 & 4289 & 136 & $8,67 \%$ \\
Estancias & 4153 & $643.705 €$ & $-205.607 €$ & $-27 \%$ \\
\hline Consumo UCI & $849.312 €$ & $885 €$ & $-384 €$ & $-24,21 \%$ \\
Coste/ingreso & $1.270 €$ & $150 €$ & $-54 €$ & $-26,26 \%$ \\
Coste/estancia & $205 €$ & & \\
\hline \hline
\end{tabular}

En las Figuras 2 y $\mathbf{3}$ podemos ver la evolución coste/estancia y coste/ingreso durante los periodos de estudio 2009 y 2010 , donde se evidencia una disminución de ambos para el periodo post-implantación. 
Fig. 2.- Coste /estancia mensual

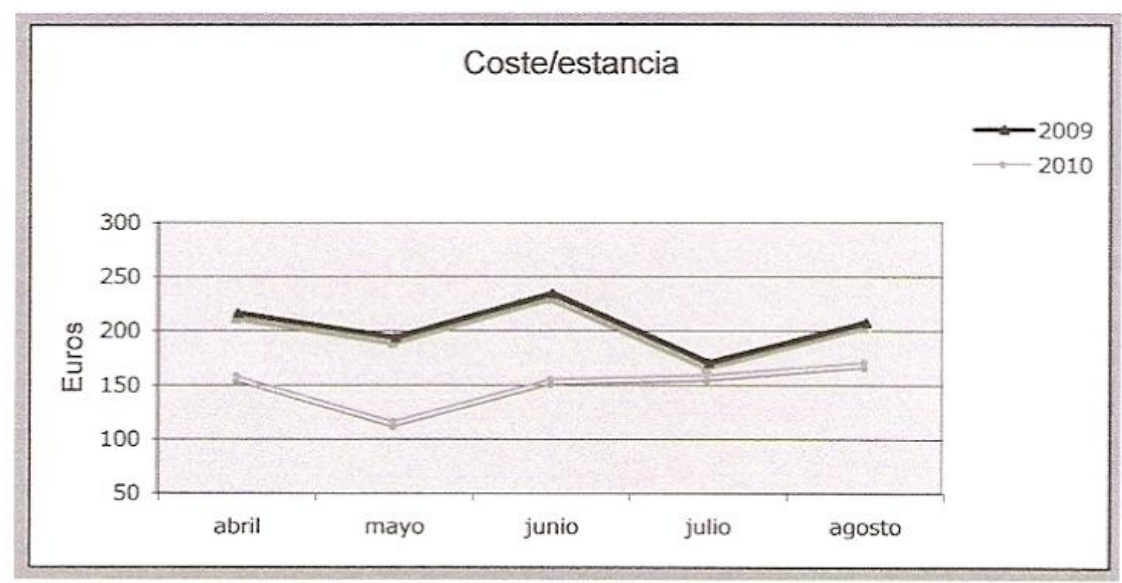

Fig. 3.- Coste /ingreso mensual

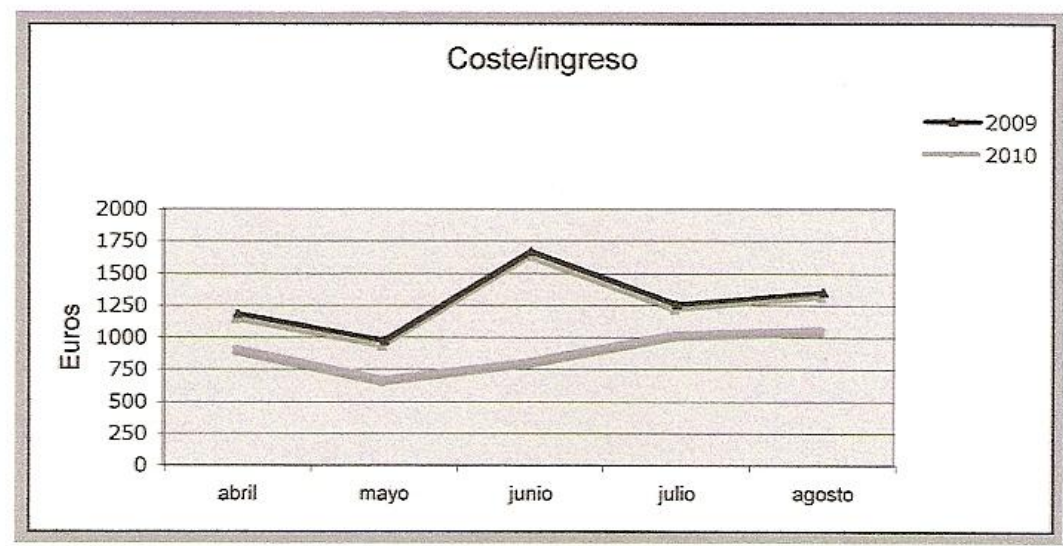

Tras su análisis, comprobamos cómo el gasto realizado en la inversión inicial de los SADME se verá rápidamente recompensado de seguir la misma tendencia, principalmente por el beneficio generado de la disminución del coste en la política de consumo de medicamentos.

\section{Satisfacción del usuario}

Se remitieron un total de 110 encuestas. La tasa media de respuesta fue del $63 \%$, siendo para el personal de enfermería del $90 \%$ y para auxiliares de enfermería del $42 \%$. El $68 \%$ de los encuestados eran mujeres y el $32 \%$ eran hombres.

La media de experiencia laboral fue de 15,73 años; encontrándose un $82 \%$ en turno de trabajo rotatorio frente al $17,3 \%$ de turno fijo. En el análisis bivariante encontramos que la satisfacción global tras la implantación de los SADME en el grupo de auxiliares de enfermería era del $58,3 \%$ frente al $54,9 \%$ en el grupo de enfermeras, no encontrándose diferencias significativas. Tampoco se encontraron diferencias al relacionar la satisfacción global con el género, años de experiencia laboral ó el turno de trabajo. 
Con respecto a si la mayoría de gente a la que respetan y admiran profesionalmente piensa que deberían utilizar los SADME, un 55\% contestaron positivamente. Un 97,3\% declaraba utilizar satisfactoriamente otros sistemas automatizados en su práctica diaria, como cajeros automáticos, máquinas dispensadoras de bebidas, tabaco, alimentos.

Sobre el control percibido por el usuario con los SADME, estimado a partir de los aspectos relacionados a su capacidad de aprendizaje y facilidad de uso, observamos que el $84,3 \%$ del personal de enfermería expresa como positiva la facilidad de uso y el $80,4 \%$ se identifican con la mayor probabilidad de aprendizaje.

En lo referente a la percepción e intención de uso de estos sistemas por parte de los usuarios reales, es de destacar que en éstos no existe correlación directa entre el esfuerzo en aprender el funcionamiento de la tecnología y los beneficios tangibles percibidos por los mismos.

Así, el 43,1\% de los enfermeros perciben una mejora con los SADME en la forma de realizar su trabajo, situación que alcanza el $62,5 \%$ en el personal auxiliar.

Sin embargo, un $80,4 \%$ del personal de enfermería contestó que sí recomendaría la implantación de los SADME en otras unidades y un 91,7\% de las auxiliares de enfermería hacían la misma afirmación.

\section{DISCUSIÓN}

Son muchos los beneficios que se le atribuyen a los sistemas automáticos de dispensación de medicamentos, tanto en la literatura como por experiencias previas, uno de los beneficios más destacados es ser una herramienta logística útil y eficaz, para el control de la utilización de los medicamentos. En este trabajo hemos querido analizar el impacto económico de la implantación de SADME en UCl y el grado de satisfacción de sus usuarios, dejando para posteriores análisis otros aspectos, también relevantes, que aportan los sistemas automáticos como son el valor añadido que la información recogida en los mismos nos aporta, conocer la farmacoterapia detallada del paciente, disminuir errores de medicación, establecer perfiles de tratamiento, desarrollar vías clínica. La información que se puede obtener de estos sistemas permite a la gerencia del hospital elaborar GRDs propios, lo que permite protocolizar el coste del tratamiento para diversos diagnósticos y optimizar la farmacoterapia.

En relación al capítulo de personal, y a los flujos que éste genera. El trabajo realizado, interpreta en términos de coste, el sistema logístico de distribución de medicamentos en su conjunto. Aunque el coste y las cargas de trabajo que se originan en farmacia, considerando este servicio aisladamente, son mayores,la realidad es que se transfieren en un porcentaje muy alto las cargas de trabajo de la Unidad Clínica al SF. Por tanto, cuando se asignan los costes a todo el proceso logístico de distribución de medicamentos en su conjunto, el hospital reduce sus costes, pero el SF aumenta los suyos. Los tiempos de personal recogidos corresponden al periodo inmediato a la puesta en marcha del proyecto, por lo que es posible que a medida que pase el tiempo y aumente el entrenamiento en las distintas funciones los costes de personal disminuyan.

En cuanto a los indicadores económicos los datos han demostrado ser muy positivos. En la misma línea, otras publicaciones han obtenido análogos índices de reducción en los costes farmacoterapeúticos en $\mathrm{UCl}^{1,2}$. Aunque su reducción porcentual esta mediatizada por la existencia previa de amplios botiquines, que sin duda han tenido repercusión en los datos 
obtenidos. Este hecho no invalida los resultados pero sí matiza la necesidad de ser prudente, a la hora de interpretar los resultados. Por ello nuestros datos, al igual que el del resto de publicaciones, pueden no tener la suficiente validez externa, incluso en hospitales de igual complejidad. Es evidente que un sistema de almacenamiento y logística anterior poco eficiente permite obtener un mayor beneficio con la implantación de los SADME.

Por tanto, los beneficios estimados en este tipo de estudios están muy relacionados con el entorno donde se desarrollan y con los procedimientos logísticos previos a la implantación que han servido para hacer el estudio comparado.

En cuanto a la valoración del personal de estos sistemas, el cuestionario nos ha permitido aproximarnos a los usuarios reales de los SADME y, en este sentido, en general, perciben una mejora en la utilización de estos sistemas como proceso logístico de distribución. Los resultados obtenidos son similares a los obtenidos en otros trabajos El control percibido por el usuario de los SADME es suficientemente consistente en magnitud como para confirmar la idoneidad del sistema, derivado del entorno "amigable" y sencillo que el software proporciona. Los datos son suficientemente consistentes como para considerar que la implantación de los SADME no ocasionará problemas con los usuarios. De hecho, una gran mayoría de los usuarios recomendarían la implantación de los sistemas automatizados a otras unidades.

\section{CONCLUSIONES}

En nuestro caso, los SADME constituyen una nueva tecnología que permiten un mayor control de los procesos logísticos relacionados con el medicamento, disminuyendo de forma considerable el gasto neto en medicamentos por ingreso y estancia, con una buena aceptación por parte de los usuarios.

\section{BIBLIOGRAFÍA}

1 Schawarz HO, Brodowy BA, Implementation and evaluation of an automated dispensing system. Am J Health-Syst Pharm 1995;52:823-8

2 Lee LW, Wellman GS, Birdwell SW, Serrín TP. Use of an automated medication storage and distribution system. Am J Hosp. Pharm 1992; 49:851-5

3 Guerrero RM, Nickamn NA,Jorgenson JA. Work activities befote an after implementation of an automated dispensing system. Am J Health-Syst Pharm 1996; 53:548-54.

4 Magnus GH. Prepared for automated dispendsing device. Am J Health-Syst Pharm 1995;52:2406-8

5 T. Bermejo Viñedo y Grupo Tecno. Papel del farmacéutico de hospital en las nuevas tecnologías en el sector sanitario. Farm. Hosp. 2010; 34(2):56-58.

6 Lacasa C, Humet C, COT R. Errores de medicación. Prevención, diagnóstico y tratamiento. Barcelona: Easo, 2000.p.241-7

7 Baker KN. Ensuring safety in the use of automated medication dispensing systems. Am J Health-Syst Pharm 1995;52:1875-9

8 L. Álvarez Rubio, J.A., Martín Conde, A., Alberdi Lens I. Evaluación de un sistema de dispensación en el Servicio de Urgencias de un hospital de tercer nivel. Farm.

Hosp.2002;27(2):72-77.

9 Pérez-Ruixo JJ, Martínez G, Quintana V, Juan J, Jiménez NV. Impacto farmacoeconómico de la implantación de un sistema de botiquín automatizado en la unidad de urgencias de un hospital general universitario. 
Farm. Hosp. 2000; 24(6):390-7.

10 Pérez-Ruixo JJ, Pastor E, Juan J, Jiménez NV. Análisis de un sistema automatizado de dispensación individualizada de medicamentos en una unidad de cuidados intensivos.

Farm. Hosp. 1998; 22:81-8.

11 [Documento en Internet ] Disponible en:

http://www.juntadeandalucia.es/servicioandaluzdesalud/library/plantillas/externa.asp?pag=/se rvicioandaluzdesalud/contenidos/profesionales/normativas/Retribuciones2009/Anexos/ANEX O I.pdf . Citado [7-02-2011 ].

12 Porta Oltra B, Gaspar Carreño ML, Pérez Ruixo JJ, Juan Colomer J, Jiménez Torres NV. Automatización en un Servicio de Urgencias pasado y presente de la dispensación de medicamentos. Farm. Hosp. 1998;22(3):129-36

13 L Poveda Andrés, C. García Gómez, M. Hernández San Salvador, A. Valladolid Walsh. Análisis coste-beneficio de medicamentos en las Unidades de Críticos y Urgencias. Farm. Hosp. 2003;27(1):4-11.

14 M. Hernández, J. L. Poveda Andrés. Sistemas Automáticos de Dispensación de Medicamentos. SEFH. Barcelona 2001 


\section{ANEXO 1}

\section{ENCUESTA SOBRE EL PERCEPCIÓN Y USO DE LOS SISTEMAS AUTOMATIZADOS DE DISPENSACIÓN DE MEDICAMENTOS (SADME)}

A continuación vas a encontrar una serie de preguntas en referencia a los Sistemas Automatizados de Dispensación de Medicamentos que hay actualmente instalados en tu servicio, POR FAVOR, responde a todas las preguntas.

Datos demográficos:
(1) Sexo: Hombre $\square$ Mujer $\square$
(2) Turno: Fijo $\square$ Rotativo
(3) Años de experiencia profesional $\square$
(4) Categoría profesional:
(5) Servicios
Auxiliar de enfermería
$\square \mathrm{UCI}$
$\square$ Enfermera/o
Otros

1.- Usar los Sistemas Automáticos de Dispensación de Medicamentos me parece: (rodea con un círculo cualquiera de los números que se encuentran entre los pares de adjetivos, dependiendo del grado de acuerdo o desacuerdo con los mismos).

$\begin{array}{lllllllll}\text { Bueno } & 1 & 2 & 3 & 4 & 5 & 6 & 7 & \text { Malo } \\ \text { Útil } & 1 & 2 & 3 & 4 & 5 & 6 & 7 & \text { Inútil } \\ \text { Interesante } & 1 & 2 & 3 & 4 & 5 & 6 & 7 & \text { Aburrido } \\ \text { Agradable } & 1 & 2 & 3 & 4 & 5 & 6 & 7 & \text { Desagradable }\end{array}$

2.- La mayoría de la gente a la que yo respeto y admiro profesionalmente piensa que deberíamos utilizar los Sistemas Automáticos de Dispensación de Medicamentos. (Rodea con un círculo el número que mejor describa la intensidad de tu opinión con respecto a la pregunta).

$\begin{array}{lllllllll}\text { Probable } & 1 & 2 & 3 & 4 & 5 & 6 & 7 & \text { Nada probable }\end{array}$

3.-La utilización de sistemas automáticos en diferentes prácticas diarias como son cajeros automáticos, máquinas dispensadoras de bebidas, comida, tabaco han supuesto una experiencia: (Marca con una cruz la respuesta elegida).

Positiva. $\quad \square$ Indiferente. $\quad \square$ Negativa.

4.- Si yo quisiera aprender a manejar los Sistemas Automatizados de Dispensación de Medicamentos en un par de semanas perfectamente lo conseguiría sin la más mínima duda: (Rodea con un círculo el número que mejor describa la intensidad con la que apoyas dicha afirmación).

$$
\begin{array}{llllllllll}
\text { Muy probable } & 1 & 2 & 3 & 4 & 5 & 6 & 7 & \text { Nada probable }
\end{array}
$$


5.- Para mí, usar los Sistemas Automatizados de Dispensación de Medicamentos en las próximas semanas va a ser... (Rodea con un círculo el número que mejor describa la intensidad con la que apoyas dicha afirmación).

$\begin{array}{lllllllll}\text { Muy fácil } & 1 & 2 & 3 & 4 & 5 & 6 & 7 & \text { Muy difícil }\end{array}$

6.- Creo que los Sistemas Automatizados de Dispensación de Medicamentos pueden ofrecer una mejora en la forma de mi trabajo.

$\begin{array}{llllllllll}\text { Muy en desacuerdo } & 1 & 2 & 3 & 4 & 5 & 6 & 7 & \text { Muy de acuerdo }\end{array}$

7.- Recomendaría la implantación de los Sistemas Automatizados de Dispensación de Medicamentos a otras unidades (Marca con una cruz la respuesta elegida).
Si.
NC
$\square$ No 